\title{
El Trabajo y el Territorio en un Mundo Globalizado(*)
}

Josep-Antoni Ybarra

Universidad de Alicante

Palabras clave: globalizacion/ desarrollo local pymes/capital socialacción institucional

RESUMEN:

Frente a los retos que supone la globalización para las economias y las culturas locales, el autor nos presenta una serie de claves para afrontar esos retos a partir de la puesta en valor del patrimonio fisico y cultural local, de las iniciativas de las entidades públicas y privadas, de la generación de capital social, etc., y siempre estableciendo redes de cooperación horizontal para buir del aislacionismo.

Key words: Globalisation, Local Development, SME's, Social Capital, Institutional Actions

\section{ABSTRACT:}

The globalisation process is a big challenge to local economies and cultures. This article exposes different key actions to face these challenges such as: the promotion of Cultural and Material Heritage value; the development of initiatives of public and private organizations; the generation of social capital, etc. Also, it is pointed out the necessity of establishing borizontal and cooperative networks avoiding isolation

(") En diferentes versiones este trabajo se ha enriquecido con las sugerencias de los asistentes al III Encuentro Comarcal por el Empleo en l'Horta Sud, Florida, Centre de Formació, Catarroja, Valencia 8-9, noviembre, 2001, Consorcio del Acuerdo Comarcal por el Empleo en l'Horta Sud y en el curso El capital social: conocimiento de regiones y países, en XXI Cursos de Verano de la Universidad de Pais Vasco, San Sebastian, 28-30 de Agosto de 2002. 
La globalización desencadena una reacción de homogeneización en cuanto a las. actuaciones sociales, económicas y productivas. Ello comporta la anulación de las capacidades y de las identidades de muchas regiones y de muchas formas de proceder: del individuo. Frente a elle, la estrategia de supervivencia que se plantea para regiones y empresas de mediano y de pequeño tamaño, es la de potenciar los recursos y el saber-hacer que sean propios. Reflexionar sobre algunas claves que permitan el poner en práctica esta estrategia será positivo. Esta estrategia girará en torno de la cooperación entre pymes y territorios, el consenso entre lo público y lo privado, la potenciación de servicios reales de carácter territorial para las empresas, la identificación de las convenciones locales que permitan un mejor funcionamiento: del mercado de trabajo y de capitales locales, y ya por fin, el generar una imagen interna y externa a los territorios que permita el valorizar positivamente aquellas: capacidades que se tienen.

1. Estrateglas de Supervivencia ante lo Global: lo local y la Pyme

La globalización no solo es un concepto que manifiesta la capacidad de interrelación del capital en el ámbito planetario (Castells, 1997); su alcance es muchø más amplio. El impacto de la globalización afecta al comportamiento de los individuos en sus manifestaciones más elementales como pueden ser en sus hábitos gastronómicos o en la indumentaria que visten. La homogeneización sobre el comportamiento de los sujetos, la uniformidad en las formas de actuar, de pensar, de interpretar suele ser la consecuencia más directa de esta manera casi irremediable de afrontar la realidad que nos inunda.

En consecuencia ante la globalización cabe preguntarse ¿y qué pasa con el trabajo?, cy con la actividad de las gentes y de los pueblos? La respuesta parece única e irrevocable: las gentes, sus actividades, sus trabajos, ..... deberán adaptarse a esta manera única de entender el mundo y de actuar. La globalización económica tiene una dinámica unívoca y tendente a la universalización (Hardt y Negri, 2002). Todo aquel comportamiento que no responda a los supuestos de rentabilidad, competitividad, mercado, precios, competencias, individualismo, agresividad, o nuevas tecnologías, serán valores obsoletos, sin futuro, arcaicos: y reaccionarios, llegando incluso a ser calificados como de antisociales (Stiglitz, 2002). Aparentemente todo lo que no sea seguir a esa tendencia única aparece como marginal, ineficiente e inapropiado. El dilema parece bien palpable: o se está con la dinámica de la globalización o se está condenado al ostracismo en cualquier campo de la actividad. económica.

El impacto de todo ello se está viendo en aspectos muy específicos como el abandono de tierras y líneas de trabajo, la polarización de la población, el cuestionar las políticașít sociales y la irrupción de los valores de mercado -precio y rentabilidad económica- en. cualquier manifestación. 
Sin embargo ante la globalización, ante la anulación del individuo, existen otras alternativas. Estas otras opciones giran en torno de la potenciación del individuo como sujeto social, en sus experiencias, en sus conocimientos, en sus territorios, en sus actividades, si bien es cierto que dotándose de una estrategia con relación a dos frentes: el territorio y la empresa, o si se quiere en lo local y en la pyme, como lugares más próximos de actuación económica. La alternativa que tienen tanto los territorios nô centrales, de dimensión intermedia, así como las pymes, es moverse en segmento de mercados concretos y específicos que sean posibles de dominar por sus especificidades. Las regiones y las pymes deben especializarse en aquello que realmente conocen, su saber-hacer específico debe de sobresalir, y aquí es donde prevalecen los aspectos relativos al trabajo y al conocimiento del proceso productivo y del producto.

Cabe advertir que la dimensión nunca puede ser el problema; la dificultad reside en la soledad, en el actuar solo. La empresa y la localidad, la pyme y el municipio que pretendan desenvolverse desde su sola y única capacidad en un marco de interrelaciones globales, están abocadas al fracaso; en el ámbito de la competencia y del mercado, la gran corporación industrial y los espacios centrales siempre encontrarán mayores ventajas que la que tiene la pyme y el municipio aislado (Benko y Lipietz, 1992). Sin embargo, cuando la pyme opera en un marco de "sistema de pyme", y el municipio actúa atendiendo a un territorio más amplio del que sus límites le han reducido, la potencialidad de estas pymes y de estos territorios es muy grande. La supervivencia local y la vitalidad empresarial hay que situarlas entonces en el terreno de la cooperación. Mediante la cooperación se alcanzan aquellas escalas que resultan necesarias para poder desarrollar procesos en los que pueden ser competitivos. Esta cooperación exigiría que aparecieran redes de regiones, redes de ciudades y redes de pymes, dispuestas a cooperar. Esta disposición sería una ventaja frente a aquellas pymes y territorios que no lo hicieran (Malecki y Tootle, 1996).

Pero ¿qué potenciar del territorio y de la localidad para no ser abolido por la presión de los grandes centros poblacionales?, ¿qué tiene un sistema pyme para afrontar con éxito el desafío al que le somete la gran corporación industrial?, ¿cómo funciona el mercado de trabajo en un sistema local de pymes?

\section{Elementos a Valorar en el Territorio y en la Pyme}

El territorio es una variable económica que va a permitir alcanzar niveles de diferenciación para las empresas que se sitúen en él. Para ello es la especificidad territorial lo que se valora en términos generales, entendiendo como especificidad territorial al conjunto de aspectos demográficos, históricos, sociales, económicos, medioambientales, tecnológicos e institucionales que se tengan; estas variables son diferentes entre unos espacios y otros, y precisamente son la base sobre la que se levantan las estructuras que permiten alzarse las sociedades y las empresas con cierto grado de capacidad y autonomía en el marco de la globalización. 
Por su parte lo que se pueden entender como especificidades económicas territoriales son un conjunto de elementos que en diferente grado van a permitir el llevar a cabo actividades económicas que partiendo de bases tradicionales tienen una capacidad de adaptabilidad a las coordenadas internacionales que les hace se: competitivas en el nuevo marco de competencias mundiales. ¿Cuáles son estos factores? ise es consciente, por parte de los individuos, de las empresas, de los sujetos intervinientes en las actividades en general, que el desarrollo de las actividades tiene unas ventajas por estar en ese territorio específico? (.por estar ligado al territorio)..

Estas variables territoriales que tienen un carácter colectivo pueden ser de res tipos:

1. Objetivas en el sentido que desde un punto de vista general se pueden deteminas Así tendriamos aspectos relativos a infraestructuras colectivas (suelo industrial, equipamientos de comunicación), o formativas (escuelas especializadas), etc.

2. Subjetivas, referidas a elementos que siendo colectivos no pueden determinarsé y trasladarse, sino que existen en el medio-territorio específico. De esta forma significa: que el estar en el medio aporta unas ventajas añadidas que de no ser así no se tendrían (ej.: el saber colectivo, la capacidad de crédito que da la organización familiar, etc) ,

3. Estratégicas, en alusión a aquellas variables que se han ido articulando de acuerdo con una política determinada hasta conformar una estrategia para alcanzar: una competitividad determinada del territorio. Podría ser una simbiosis de las dos anteriores trabajadas de forma sistemática (ej. la creación de un Instituto Tecnológico o un centro de servicios avanzados para la empresa).

Como elementos a destacar en este conjunto de variables territoriales sobresalen bas convenciones locales que permiten el desarrollo especifico. Estas convenciones son aquellas: normas y reglas de carácter "extramercado" y "extrainstitucionales" que posibilitan que se alcancen unos determinados umbrales de desarrollo a todos los niveles en el territorio concreto en el que se manifiestan. Son las normas económicas socialmente aceptadas pófla comunidad local, reafirmadas por el uso y la costumbre comunes ampliamente ratificadss por la tradición en el lugar de que se trate y que permiten que la comunidad sancione o: permite a los sujetos en tanto que se cumplan (Coleman, 2001; Woolcock, 1998).

En el marco de estas convenciones especificas podemos referimos a aspectos comid la cultura empresarial, movilidad social, capital territorial, relaciones interempresariales; cooperación y colaboración, saber colectivo, o enseñanza y formación en el medio. Todos ellos permiten que las economías del lugar, las ventajas de situarse en un higa específico que posea unas convenciones determinadas hará que se alcaneẹ posibilidades de llevar a cabo actividades que en otros lugares no se tienen.

Situándonos en el plano de los factores de producción, la accesibilidad que se tiene al trabajo o al capital va a ser diferente en tanto que se haga atendiendo a consideraciones territoriales y locales. En un distrito incustrial o en un sistema produgtipi local (SPL) la seguridad y la confianza que tienen los sujetos económicos en cómojs 
accede a estos factores es mucho mayor que si se tratase de espacios más anónimos y generales. De la misma forma, la seguridad en cómo los oferentes de capital y trabajo hacen al conjunto del sistema local es mucho mayor que en otros lugares que no reúnan las características locales. Entonces las funciones de la banca local y de la familia en cuanto a proveedores de capital tienen unas peculiaridades específicas que difieren de las que pueden tener en otro tipo de sistemas y economías (Portes, 1998).

Otro tanto cabe advertir al considerar los aspectos relativos al trabajo en el sentido que:

1. Existe una sensación de "Confianza y seguridad" en el SPL, el distrito o el territorio productivo que se trate, cuando se va en la búsqueda de trabajo. De hecho se llega a la convicción de que es más sencillo encontrar trabajo en un SPL que en otro lugar. Posteriormente también se comprueba que el SPL es más seguro para la continuidad en el trabajo.

2. Cabe apreciar que los trabajadores-asalariados no lo son tanto cle una empresa sino que pueden ser calificados como trabajadores del territorio. Puede existir movilidad interempresarial en cuanto a los trabajadores que sean contratados por una $u$ otra empresa, pero difícilmente esos trabajadores saldrán del territorio en cuestión para ser contratados.

3. Lo anteriòr imprime una característica específica al trabajo en el SPL, y es que el trabajo se considera como un bien social-colectivo. Se entiende el trabajo en los SPL como un término genérico, pero que encierra elementos concretos para su desarrollo como son, la experiencia, el saber-hacer, el conocimiento específico acumulado, la versatilidad, la flexibilidad para adaptarse a las necesidades especiales que el territorio puede precisar. Esto es, el trabajo en los SPL posee unas características diferenciales de lo que puede ser ese mismo trabajo en otros espacios.

4. De entre las características relevantes que tiene el mercado de trabajo en territorios específicos sobresale la cualificación de la mano de obra. Esta capacitación de la mano de obra es específica para hacer frente a las necesidades concretas que el SPL puede tener. Esta capacitación además suele ser colectiva y general para el conjunto de la mano de obra del SPL. Ello imprime una ventaja importante al territorio, en tanto que la cualificación de la mano de obra y su generalización permite alcanzar una competitividad al espacio que otro no posee. Puede calificarse entonces la cualificación generalizada de la mano de obra como un bien colectivo de uso territorial.

5. Específicamente este bien colectivo que es el trabajo cualificado existente en un SPL tiene una especificidad que imprime una ventaja adicional referida a la transmisión del conocimiento y a la forma cómo se traspasa el saber-hacer. El conocimiento productivo es un conocimiento social y colectivo de carácter territorial; la transmisión de este conocimiento es igualmente social y colectiva adherida al territorio. Esta transferencia no requiere formas estructuradas y estandarizadas para su comunicación -escritas la mayoría de las veces-; incluso cabría pensar que su 
estandarización en cuanto a la forma de transmisión es imposible o al menos muy cuestionada. Es la costumbre, el uso continuado, los cambios consecutivos, las experiencias acumuladas, la cuotinianidad social lo que hace que se conozca el hacer, los problemas, las soluciones, las peculiaridades que tiene un procesø productivo. Ese saber-hacer no está escrito sino en el ambiente, y es transferido: por la práctica con la ventaja adicional de su adaptabilidad.

6. En los mercados de trabajo locales existe una forma específica de establecer las relaciones de trabajo. Son las relaciones personales, amistosas y directas las que priman sobre cualquier otra. De aquí que las relaciones laborales de producción estén condicionadas por estas relaciones personales. Ello singulariza las formas de: comportamiento en el mundo del trabajo en cuanto a exigencias, sumisión, horarios, salarios, profesionalidad, condiciones, etc. Las relaciones técnico-productivas están: supeditadas a las relaciones paternalistas. Sin embargo, ello no significa que la profesionalidad esté cuestionada; muy posiblemente si la cualificación de un trabajador no fuese la mínimamente exigida para su puesto de trabajo, a pesar de sus posibles relaciones amistosas con su contratista, este trabajador no seria contratado. En cambio, debido a las relaciones paternalistas, otras condiciones: que no se cumplan (en cuanto a horarios más dilatados, sistemas de trabajo más: exigentes, impagos de ciertas prestaciones -vacaciones, horas extraordinarias: etc.) no serían reclamadas.

7. La anterior característica de los mercados locales de trabajo cuestiona la capacidad de las instituciones vigilantes del funcionamiento de este mercado. En concreto los sindicatos estarán en entredicho en estos mercados, al menos en sus formas más tradicionales y generales de funcionar: firma de convenios colectivos $\mathbf{y}$ vigilancia en su cumplimiento, normas en cuanto a salubridad e higiene en el trabajo, etc. Sin embargo, si estas instituciones no pueden hacer que se cumpla en su completa rigurosidad los acuerdos que se hayan establecido con carácter general, sí que van a hacer que se adapten los acuerdos generales a la especificidad local de que se trate; así aparecen cuestiones como ubolsa de horas local", adaptaciốn de vacaciones a los compromisos productivos locales, etc. Algo parecido ocurre, 0 puede ocurrir, con la banca, con seguros, con la educación o con muchas otras instituciones locales; estas instituciones se adaptan a las especificidades territoriales:

8. El mercado local de trabajo tiene una característica que otrós mercados dé trabajo no tienen y es la posibilidad de dar una movilidad social que en otras circunstancias (territoriales y sectoriales) serían muy difícil de tener. Ello da unä confianza y expectativas al trabajador cualificado considerable.

9. Los mercados de trabajo locales transfieren una sensación de confianza đụe otros mercados tampoco poseen. Confianza que se concreta a niveles personalies y profesionales en cuanto a quién contrata, qué contrata, cómo contrata, etc. Pố su parte el contratista conoce el saber-hacer, la profesionalidad, dẹl contratádo. 
10. Los mercados de trabajo locales están condicionados a las relaciones familiares. La familia representa un lugar central como unidad no solo de consumo sino fundamentalmente como unidad de producción, como unidad de seguridad, de apoyo, de confianza, de traslado de experiencias, etc.

Todo ello permite que podamos afirmar que el capital social acumulado en un SPL $O$ en distrito industrial es muy grande y tiene características y peculiaridades específicas y no alcanzadas en otros lugares. Pero subrayamos qué es capital social territorial. Hay que entender que son temas específicos pero de difícil cuantificación económica relativos al saber-hacer, a la transmisión de conocimientos, al acceso al capital financiero, al conocimiento de temas específicos, etc. (Putnam, 2001 a; Woolcock, 2001) Entonces la capacidad de cambio y la capacidad de desarrollo de un SPL o de un distrito industrial va a depender de la cantidad y calidad de capital social que tenga acumulado.

El esquema clásico que la Economía Política tiene para analizar la dinámica y el crecimiento económico es preguntándose por la fuente del beneficio en tanto que ello va a permitir conocer las posibilidades de acumulación y en algún sentido las de inversión, que son las que en definitiva permitirian hablar de crecimiento. La secuencia es: beneficios > cumulación > inversión > crecimiento. Sin embargo, con la figura del distrito industrial y de los SPL se requiere que debamos interponer en nuestros esquemas interpretativos la idea de que el mecanismo de crecimiento está modificado por la acumulación de capital territorial que el distrito posee (McNaughton, 2000). Ello hace que el esquema secuencial sea diferente porque si bien es cierto que en el proceso de desarrollo clásico los hicrementos de productividad se derivan de los incrementos de productividad del trabajo o del capital, hasta hacer que se produzca un proceso de acumulación yque, en algunos casos, definitivamente comporte progreso y bienestar. El tema es que los incrementos de productividad en los distritos industriales son debidos a la utilización del capital social acumulado al que cualquier sujeto situado en ese espacio puede acceder. El propio distrito es un hacedor de los incrementos de productividad del capital y del trabajo, y por tanto susceptible de incrementar sus posibilidades de crecimiento. El tema se concreta entonces en cómo hacer para aumentar el capital social territorial.

\section{La Política para el Desarrollo del Capital Social Territorial}

La cantidad y calidad del capital social acumulado va a depender de dos cosas; en primer lugar del volumen de las convenciones que existan en el territorio en Gurestión; y en segundo lugar de los niveles de equipamiento colectivo de carácter econónico que se tengan. Tanto los unos como los otros son susceptibles de aumentarse con la salvedad de que llegan a ser dependientes entre sí. Los niveles de 
equipamiento social de carácter económico tales como los institutos tecnológicos, los centros de demostración, las agencias de desarrollo local (ADLs), etc. van a determinar la cultura empresarial que se tenga, la calidad de las relaciones industriales; la confianza en la solicitud de un préstamo, etc. Y por su parte esa cultura industrial territorial, las variables extramercado, la confianza y la colaboración que existan en el territorio, es decir las convenciones territoriales, harán que vayan apareciendo equipamientos de tipo formativos, demostrativo, de homologación, etc. propios de territorios económicos fuertes.

Entonces cabría preguntarse por el quién, el cómo y el por qué debe hacerse para dotar al territorio de un mayor y mejor capital social. El debate está servido en tanto que se pretenda responder a cualquiera de las preguntas anteriores.

A modo de cuestión ejemplar vamos a centrarnos en las $\mathrm{ADL}$, pero que pueden ser ampliado en el razonamiento de cualesquier otros equipamientos territoriales que se quieran plantear. Así, quien quiera que haya profundizado en las razones y en las actuaciones de las ADLs siempre va a encontrarse ante la misma polémica: el sector público entiende que son una herramienta necesaria para dinamizar el ámbito local en el que se ubican, mientras que el sector privado entiende que las ADLs están invadiendo parcelas que no le corresponden, haciéndolo además con capitales de la colectividad. La discusión en el fondo siempre es la misma: ¿cuál es el ámbito y el límite de actuación del sector público? ¿tienen sentido la política pública de carácter local?

La controversia que existe entre el ámbito público y el privado no deja de ser una polémica interesada y siempre partidista. Así la voluntad pública de dotar al territorio de instrumentos capaces de elevar la competitividad del territorio y de explicitar la potencialidad del lugar, siempre será criticada por aquellos sujetos que de forma autónoma tengan la capacidad financiera o la posibilidad técnica de llevar a cabo estas mismas actuaciones. Sus campos de trabajo pueden ser coincidentes y por ende las críticas. Sin embargo cabría preguntarse, esas dotaciones que tiene una empresa particular, ¿pueden ser utilizadas por el resto de empresas del territorio en cuestión? La respuesta dependerá del grado de interés que la empresa que posea las dotaciones en cuestión tenga en monopolizarlas o no. Entonces, la actuación pública tendrá como objetivo el ofertar a la colectividad aquellos servicios que pueden ser útiles con carácter general, teniendo además la característica de poder dotar de aquellos servicios que aún sin ser rentables a título individual, sí pueden serlo para la comunidad (entendemos aquí servicios que pueden desconocerse o tener una dimensión excesivamente grande).

Esta polémica se enlaza con otra que trata de profundizar en la nueva forma de hacer política en donde la discusión, política pública versus privada, tiene poco sentido; es una discusión del siglo pasado. Hoy de lo que se trata es de conformar una nueva forma de hacer política en donde la colaboración prevalece por encima de todo; advirtiendo además otra cosa, que el sector público tiene una obligación por encina 
del sector privado y es estar siempre un paso por delante de lo que el mercado está exigiendo. Adviértese que el mercado es solo una forma de asiignar recursos, pero pueden existir otros mecanismos en el territorio tan eficientes o más que el mercado y que deben tenerse presente. Piénsese que la valoración que en términos de precios puede hacer el mercado sobre el conocimiento colectivo, el saber-hacer, la trasferencia de conocimientos, la dotación de créditos a miembros de una familia, la iniciativa privada, las interrelaciones empresariales, etc., son simplemente nulas. La colaboración entre lo público y lo privado debe prevalecer sobre la situación de confrontación que podría primar en otras circunstancias. El llegar a concretar compromisos y contratos entre ambas partes sería un avance sustancial. Nueva forma de hacer política ante los huevos problemas (Putnam, 2001 b).

Incluso en el ámbito europeo, el replantearse la política industrial va en el sentido que estamos apuntando. La política de apoyo a la empresa individual o al sector específico ya no va siendo posible, sino que lo que se va a plantear son políticas en el ámbito colectivo. Y aquí es donde tiene sentido hablar de política territorial de carácter industrial, o industrial de carácter territorial; trabajar con los distritos industriales o los SPL. Así lo què se plantea es hacer política para dotarse de una inteligencia colectiva en el SPL, de un capital social colectivo de ámbito territorial (Glaeser, 2001).

En concreto aparecen los pactos territoriales como los grandes instrumentos para la acción territorial si bien muchas veces están vacíos de contenido. Advertimos que estos pactos territoriales son fruto de acciones previas que desembocan en este tipo de operaciones debiendo además incluir al menos tres niveles o áreas de actuación: una primera que tenga presente los aspectos relativos a la calidad de vida, que eleve los niveles de salud, bienestar, relacionales, espacios colectivos, etc.; un segundo que se centre en la integración y el desarrollo empresarial, que persiga el incremento de las actividades económicas con repercusión en el ámbito social y productivo general; y un tercer nivel que trate de la concertación entre lo público y lo privado, que se preocupe por las nuevas formas de la gobernabilidad.

\section{Claves para la Accion Territorial y laboral en Tiempos de Globalizacion}

El diseño de acciones que tiendan a valorizar el territorio ante las presiones de la globalización homogeneizadora, se fundamenta en algo tan simple como la potenciación de los recursos locales y del entorno allá donde se desenvuelve la pyme y el individuo. El trabajo bien hecho y el saber-hacer del sujeto serán garantías para su supervivencia. Esos recursos del medio y del entorno deben entender en términos tanto económicos como no económicos atendiendo a la especificidad territorial referida al conjunto de aspectos demográficos, históricos, sociales, económicos, medioambientales, tecnológicos e institucionales que se tengan. 
La potenciación de estos recursos se acompaña de actuaciones tanto públicas como privadas que vengan a manifestar las posibilidades de los territorios en cuestión. advirtiendo además que deben paliarse aquellas necesidades que para su normal desenvolvimiento se tuviesen. Las claves del normal desenvolvimiento al que nos referimos se encontrarían en:

1. Enfrentarse a la soledad, vulnerar el aislacionismo. Plantear la cooperación como algo intrínseco para la supervivencia en tiempos de globalización. Entiéndase que la cooperación no es un acto altruista y voluntarioso, sino una obligación derivada de la necesidad. Esta se daría tanto entre empresas, creando sistemas pyme, como entre territorios, hasta alcanzar dimensiones operativas para poder hacer frente a las posibles ineficacias que se manifiestan por pymes y territorios aislados ante la gran empresa transnacional y los grandes centros poblacionales.

2. Modificar las formas de hacer política en dos sentidos. Por un lado en tratar de aproximar los resultados a los programas, y por otro el resaltar la necesidad de alcanzar compromisos entre las acciones públicas y las privadas. Las formas de concretar estos compromisos y acuerdos se pueden decidir en contratos y normas, las cuales se deberán fiscalizar pidiéndose responsabilidades.

3. Potenciar los servicios reales que se requieran en el ámbito de territorio y de empresas hasta dotarlos de equipamientos productivos que por sus dimensiones las empresas no tendrían. En el marco de potenciar la especificidad encontramos aspectos que difícilmente pueden ser alcanzables por una empresa a título individual y que sin embargo son necesarios para su mejor desenvolvimiento e incluso para el desenvolvimiento de la colectividad. Unas veces esta imposibilidad será de carácter financiero porque una sola empresa no pueda costear por sí sola (como pueda ser el realizar una carretera que permita una mayor accesibilidad para sus productos), otras será de carácter técnico (porque no tenga capacidad de investigación en determinados aspectos tecnológicos) o simplemente la incapacidad aparecerá por ignorancia (por el hecho del desconocimiento que pueda tener de determinados productos o servicios). Esto hace que aparezca la necesidad de potenciar una serie de servicios reales que siendo necesarios para la mejora en el desarrollo de las empresas, estas empresas no tienen acceso por falta de capacidad financiera para acceder a ellos, o por no existir en el mercado. En tanto que aparezca una mayor oferta de servicios reales para las pymes, mayor será su capacidad de competencia. Esta será una de las políticas claramenté diferenciadoras entre territorios y empresas.

4. Necesidad de explicitar las peculiaridades de los mercados de trabajo locales y de las formas de acceder al mercado de capitales que se tienen específicamente en $10 \mathrm{~s}$ ámbitos locales. El alcanzar compromisos tanto implícitos como explícitos de caráctê particular en temas relativos al funcionamiento del mercado de trabajo como en ef acceso al capital es una de las cuestiones concretas que se aprecian en los tentitorios Ias: 
convenciones -los acuerdos extramercado- es la ligazón que da naturaleza al territorio y posibilita el que este territorio sea capaz de desarrollarse atendiendo a sus capacidades. Sacar a la luz, profundizar y mejorar el funcionamiento de estas convenciones locales sería un requisito substancial para poder alcanzar niveles de desarrollo local.

5. La creación de una imagen que identifique tanto interior como exteriormente al territorio productivo será un último requisito para el desarrollo. Esta imagen debe concretar el saber-hacer local, la potencialidad de sus recursos, la calidad de sus productos, la capacidad de sus gentes. La elaboración de un Plan Estratégico que incluya el marketing territorial pasa a ser una condición más para el desarrollo. El orgullo local por su saber-hacer debe trasladarse en términos concretos hacia el exterior. Así desde la creación de centros de demostración hasta el cuidado por la imagen medioambiental, serán elementos a valorar en una estrategia de desarrollo territorial.

\section{Bibliografía}

Benko, G.; A. Lipietz (edits) (1992), Les régions qui gagnent, Paris: PUF.

Castells, M. (1997), Economía, Sociedad y Cultura. La era de la información. La sociedad red, vol. 1, Barcelona: Alianza Editorial.

Coleman, James S. (2001), "Capital social y creación de capital humano", Zona Abierta, 94/95: 47-81 (traduc. de American Journal of Sociology, 1988, 94: 95-120).

Glaeser, E. (2001), "La formation du capital social", ISUMA, Revue Cannadienne de Recherche sur les Politiques, vol. 2, 1, printemps, pp: 38-45.

Hardt, M.; A. Negri (2002), Imperio, Barcelona: Paidós.

Malecki, E.; D. Tootle (1996), "The role of networks in small firms competitiveness", International Journal of Technology Management, vol. 12, 1/2, pp: 43-57.

McNaughton, R.B. (2000), "Industrial Districts and Social Capital", in M.B. Green, R.B. McNaughton, Industrial Networks and Proximity, Hampshire: Ashgate Publishing, pp: 69-85.

Portes, A. (1998), "Social capital: its origins and applications in modern sociology", Annual Review of Social Sciences, vol. 24, pp: 1-24.

Putnam, R. (2001 a), "Mesure et conséquences du capital social", ISUMA, Revue Cannadienne de Recherche sur les Politiques, vol. 2, 1, printemps, pp: 47-58.

Putnam, R.D. (2001 b), "La comunidad próspera. El capital social y la vida pública", Zona Abierta, 94/95: 89-104.

Stiglitz, J.E. (2002), El malestar en la globalización, Madrid: Taurus.

Woolcock, M. (1998), "Social capital and economic development: Toward a theoretical sinthesis and policy framework", Theory and Society, 27: 151-208.

Woolcock, M. (2001), "Le rôle du capital social dans la compréhension des résultats sociaux et économique", ISUMA, Revue Cannadienne de Recherche sur les Politiques, vol. 2, 1, printemps, pp: 11-18. 